\title{
Lower urinary tract symptoms in men: A case-based discussion
}

\author{
Greg Bailly, MD, FRCSC,; J. Curtis Nickel, MD, FRCSC ${ }^{+}$
}

*Associate Professor and Residency Program Director, Department of Urology, Dalhousie University, Halifax, NS; † Professor, Department of Urology, Queen's University and Tier 1 Canadian Institute of Health Research (CIHR) Canada Research Chair in Urologic Pain and Inflammation, Kingston, ON

Cite as: Can Urol Assoc J 2012;6(5):S146-7. http://dx.doi.org/10.5489/cuaj.12206

\section{Abstract}

At the conclusion of the sessions on lower urinary tract symptoms (LUTS) in men, participants at the 2012 Canadian Urology Forum (academic and clinical urologists from across Canada) engaged in a discussion of optimal LUTS evaluation and management. The discussion was led by Dr. J. Curtis Nickel and was based on a patient case. This review provides a summary of the case and the discussion it generated.

\section{Case study: Presentation}

A 58-year-old man presented with gradually increasing lower urinary tract symptoms (LUTS) that had evolved to the point of being bothersome enough to consult his physician.

His symptoms included hesitancy, slow stream, moderate urinary frequency, nocturia two times per night, a feeling of incomplete bladder emptying, stranguria, perineal discomfort and deep pelvic pain lasting for several hours after ejaculation.

Physical examination did not reveal any obvious bladder distension and his prostate was moderately enlarged and benign. Light palpation of the prostate exacerbated the pelvic and perineal pain.

Urinalysis was normal and prostatic-specific antigen (PSA) was reported as $1.2 \mathrm{ng} / \mathrm{mL}$. He was on no medication for this problem.

\section{Discussion}

The participants at the 2012 Canadian Urology Forum were asked what further testing they would order at this point. The multiplechoice answer selected by the largest bloc of participants (50\%) was urine culture, uroflow and post-void residual (PVR). The session moderator, Dr. J. Curtis Nickel, agreed that this was a reasonable selection, but suggested that the urine culture should be gathered before and after prostate massage and that uroflow may not be necessary at this juncture.

\section{Case study: Initial management}

At the time of presentation, a PVR was conducted and the volume was found to be $80 \mathrm{~mL}$. Urine cultures were sent to the laboratory.

The Forum participants were asked at this point whether or not they would initiate pharmacotherapy while awaiting the results of the urine culture. Approximately three-quarters of the participants said they would initiate therapy with an alpha-blocker, either alone $(37 \%)$ or in combination with an antibiotic (32\%) or with a 5-alpha reductase inhibitor (5\%). A further $21 \%$ of respondents indicated they would initiate therapy with an antibiotic alone, while only $5 \%$ would await the results of testing before recommending any treatment.

In the actual case, the physician elected to treat with a fluoroquinolone antibiotic before obtaining the results from the urine culture.

\section{Case study: Follow-up, additional testing and changes in therapy}

The patient reported that he initially believed he was getting better, but after two weeks his symptoms were back to baseline. The urine cultures turned out to be sterile.

The participants were then asked what further testing they would order at this point. Forty-one per cent responded that they would order uroflow and cystoscopy, $12 \%$ would order uroflow alone and a further $12 \%$ would order a cystoscopy with urodynamics. Meanwhile, 35\% of the respondents said they would initiate another treatment at this time.

In the actual case, the physician elected to order uroflowmetry, which revealed a maximum flow rate of $12 \mathrm{~mL}$ per second, with a voided volume of $350 \mathrm{~mL}$. The participants were asked what course of action they would take now that they have this information. Half of the respondents indicated they would try an alpha-blocker alone, one-third would initiate an alpha-blocker in combination with an anti-inflammatory, $11 \%$ would recommend triple therapy with an alpha-blocker, anti-inflammatory and 5-alpha reductase inhibitor, and $6 \%$ would initiate neuromodulatory therapy (e.g., tricyclic antidepressant and/or gabapentinoid). 
The physician in the actual case elected to use the alphablocker/anti-inflammatory combination. However, the patient did not respond to this strategy. When asked if they would seek any additional information from diagnostic testing, almost all of the participants selected cystoscopy, either with (44\% of respondents) or without $(50 \%)$ video-urodynamics. Six per cent said that they would like to see the results of an MRI or CT scan. The physician in the case ordered cystoscopy, which disclosed moderate lateral lobe prostate hyperplasia and a high riding "tight" bladder neck with no significant median lobe. Videourodynamics were also performed; this showed high voiding pressures with a poorly opening bladder neck.

The final question for this patient was whether or not surgery should be considered and, if so, which procedure might be the best fit for this patient. Most participants (88\%) indicated that they would recommend surgery, with $46 \%$ opting for transuretheral incision of bladder neck (TUIBN), 24\% for transurethral resection of the prostate (TURP), and $18 \%$ for transurethral incision of the prostate (TUIP).

Competing interests: Dr. Bailly received compensation from Astellas realted to this article and to the Urology Forum 2012. Dr. Bailly is also a paid consultant with Allergan, Pfizer, and Eli Lilly.

Correspondence: Dr. Greg Bailly, 620-5991 Spring Garden Rd, Halifax, NS B3H 1Y4; gbailly@dal.ca; Dr. J. Curtis Nickel, Department of Urology, Victory 4 - 76 Stuart St., Kingston, ON K7L 2V7; icn@queensu.ca 